\title{
Robustness of Hopfield Neural Networks Described by Differential Algebraic Systems of Index-1 under the Conditions of Deviation Argument and Stochastic Disturbance
}

\author{
Qing Liu, ${ }^{1,2}$ Ping Li $\mathbb{D}^{1,2}$ Zuqiao Yang, ${ }^{1,2}$ and Zhibing Liu $\mathbb{i}^{1}$ \\ ${ }^{1}$ College of Mathematics and Statistics, Huanggang Normal University, Huanggang 438000, China \\ ${ }^{2}$ Hubei Provincial Key Laboratory of Metallurgical Industry Process System Science, \\ Wuhan University of Science and Technology, Wuhan 430081, China
}

Correspondence should be addressed to Ping Li; 258803198@qq.com

Received 19 September 2021; Accepted 8 November 2021; Published 29 November 2021

Academic Editor: Mehmet Emir Koksal

Copyright $\odot 2021$ Qing Liu et al. This is an open access article distributed under the Creative Commons Attribution License, which permits unrestricted use, distribution, and reproduction in any medium, provided the original work is properly cited.

Robustness refers to the ability of a system to maintain its original state under a continuous disturbance conditions. The deviation argument (DA) and stochastic disturbances (SDs) are enough to disrupt a system and keep it off course. Therefore, it is of great significance to explore the interval length of the deviation function and the intensity of noise to make a system remain exponentially stable. In this paper, the robust stability of Hopfield neural network (VPHNN) models based on differential algebraic systems (DAS) is studied for the first time. By using integral inequalities, expectation inequalities, and the basic control theory method, the upper bound of the interval of the deviation function and the noise intensity are found, and the system is guaranteed to remain exponentially stable under these disturbances. It is shown that as long as the deviation and disturbance of a system are within a certain range, there will be no unstable consequences. Finally, several simulation examples are used to verify the effectiveness of the approach and are described below.

\section{Introduction}

Life is full of nonlinear phenomena, for example, the resistance of a plane and the starting of a car. On the other hand, scholars have never stopped the comprehensive analysis of nonlinear systems and have mainly focused on system control, for example, adaptive neural finite-time stabilization [1], adaptive control [2,3], repetitive control [4], output feedback stabilization $[5,6]$, system commutativity issues [7], $H_{\infty}$ control [8], and fuzzy second-order-like sliding mode control [9]. Similar to p-normal forms, planar switched nonlinear systems, and semi-Markov jump nonlinear systems, the modeling and analysis of some nonlinear systems are very complex. The control of system transient processes and the restriction of nonlinear functions are the challenges of this study. Hence, it is meaningful to devise appropriate control methods for the study of nonlinear systems.
A deviation argument is a function that is related to a certain degree-of-deviation variable and is also a generalization of a time delay. This kind of complex system shows continuity and discreteness in the operation process. Systems with DAs are usually composed of differential equations and difference equations.

This special structure makes up for the local problems in the practical application of artificial neural networks and Biomathematics. Therefore, research on this kind of system also has been increasing. In [10], an adequate condition for the existence and uniqueness of the solution of a system was given. In [11], the control scheme of a sequence of hybrid systems was improved by using sampling data control and pulse control. In [12], the optimal control problem of a system is solved. A series of notable issues have been comprehensively analysed, including out-synchronization and matrix measure approaches for stability and synchronization [13-15]. 
Stochastic disturbances (SDs) also inevitably appear in nonlinear systems. For instance, in the transmission process of information along a channel, it inevitably interferes with various internal and external noise, which leads to the deterioration of the information transmission quality. In a radar tracking system, stochastic is embodied in the irregular movement of a tracked object and a large number of external disturbances. Obviously, nonlinear models with SDs increase the rationality of modeling the real world. However, it is very difficult to analyse and design schemes, which has always been a popular are of research. In [16], the exponential attractor discrete set (MJDS) with (SDs) was obtained by IC. It is worth noting that the subject IC condition is relatively loose. In [17], the issue of $H_{\infty}$ boundary state output feedback control with collocated boundary measurements is explored. An adaptive neural network statefeedback controller was designed by building an appropriate Lyapunov function in [18]. There are other conclusions about the systematic control design of SD systems in references $[19,20]$. Extending these approaches to DASs is a challenging topic.

It is an innovation to describe neural networks with DAE. A neural network is a typical nonlinear dynamic system that can be used to describe, recognize, and make decisions and control intelligent behaviour. The core is the cognition and simulation of intelligence. Neural networks have refreshed the concept and function of computing and made scholars have a new understanding of them. Furthermore, neural networks have a wide range of applications, such as speech recognition, image recognition, intelligent robots, and even medicine [21, 22]. Koppe et al. [23] used recurrent neural networks to evaluate the dynamics of neuroimaging data. Yuksel et al. [24] predicted the groundstate binding energies of atomic nuclei with a neural network model. Researchers are mainly interested in the dynamic evolution of neural systems. In [25, 26], fuzzy logic and neural network control problems are explored. As we know, in the early 1980s, Hopfield combined statistical mechanics with systematic learning and proposed the classical Hopfield neural network model, and this model can also be derived through microelectrical components. The feasibility of artificial neural networks was demonstrated, and then, there was an increase in qualitative research on artificial neural networks.

Although research on nonlinear systems is very mature, there are few conclusions about the robustness of disturbed systems. For example, the Lyapunov stability theorem, controller design, semigroup theorem, and linear matrix inequality (LIM) methods have difficulties achieving robustness for nonlinear systems with disturbances. Hence, there is still much room for improvement for this issue.

DASs are also known as DAEs, which are generalized equations that use differential systems to represent complex systems. The algebraic equations in DAE systems are constraints that describe actual motion through mathematical theory. Due to the application of DAEs in chemical processes [27], power systems [28], computer algebra systems [29], etc., their system control problems have received unprecedented attention. An observer in the form of a DAE is designed for nonlinear DASs and can improve system performance [30]. The sampling data controller is set with an appropriate sampling period and scale gain to guarantee the stability of the whole closed-loop system [31]. By interpreting the DASs as feedback interconnections between a pure differential equation and an algebraic equation, the stability analysis is analogous to a small gain-like condition [32]. A symplectic indirect method is proposed for the optimal control problem with index-1 DASs in [33].

The study of the stability of a class of DASs is still in its initial stage. The Lyapunov direct method and small gainlike arguments are commonly used. However, methods for DASs with deviation functions and SDs are difficult to study. When the interval length of the DA or the SDs exceeds a fixed range, the DA and SDs in a nonlinear system will cause instabilities. Therefore, the stability of a system depends on its own strength. If the influence of a deviation and disturbance is small, the system will remain stable. Therefore, the interval length of a deviation and the quantitative index of the disturbance intensity are of great significance for the study of nonlinear systems. In fact, there are few direct studies on the robustness of DASs with deviations and disturbances. In this paper, the robustness of index-1 DASs is studied by using the theory of parallel differential systems. Based on a concrete model, the upper bounds of the deviation function and disturbance are given.

The remainder of this paper is laid out as follows. Section 2 describes the origin of the model; some definitions, conditions, and lemmas are given; and the proof process of these lemmas is provided. Section 3 provides an analysis of the robustness of a system. In Section 4, two numerical examples are given. Finally, Section 5 provides a brief summary and future work directions.

\section{Preliminaries and Model Description}

2.1. Notations. The meaning of symbols will be used in the whole text. $N$ represents natural numbers, $R^{+}=[0,+\infty) . R^{n}$ is a space of $n$-dimensional real numbers. $\|\bullet\|$ is $L_{1}$ norm in $R^{n}$, as $\sum_{i=1}^{n}\left|v_{i}\right|=\|v\|_{1}$. Define two sequences of real values $\left\{\alpha_{k}\right\},\left\{\eta_{k}\right\}, k \in N$, when $k \longrightarrow+\infty$, such that $\alpha_{k}<\alpha_{k+1}, \alpha_{k} \leq \eta_{k} \leq \alpha_{k+1}$.

2.2. Model. Then the model of this paper is introduced as follows.

As we known, traditional Hopfield neural network models are usually expressed by differential equations,

$$
C_{i} \frac{\mathrm{d} x}{\mathrm{~d} t}=-\frac{x_{i}}{R_{i}}+\sum_{j=1}^{n} T_{i j} g_{j}\left(x_{j}\right)+I_{i}, i=1, \ldots, n .
$$

Among them, the resistor $R$ and capacitor $C$ formed in parallel mimic the time constant of the biological nerve output, and the transconductance $T$ simulates the synaptic characteristics of the interconnection between neurons.

When capacitor $C$ is broken down, this phenomenon can be expressed by the following static equation: 


$$
0=-\frac{y_{i}}{R_{i}^{\prime}}+\sum_{j=1}^{n} T_{i j}^{\prime} g_{j}^{\prime}\left(y_{j}\right)+J_{i}, i=1, \ldots, n
$$

The neural network model described by DAS is as follows:

$$
\left\{\begin{array}{l}
\frac{\mathrm{d} x_{i}(t)}{\mathrm{d} t}=-c_{i} x_{i}(t)+\sum_{j=1}^{n} a_{i j} f_{j}\left(x_{j}(t)\right)+\sum_{j=1}^{n} b_{i j} g_{j}\left(y_{j}(t)\right) \\
+I_{i} \\
0=-d_{i} y_{i}(t)+\sum_{j=1}^{n} p_{i j} h_{j}\left(x_{j}(t)\right)+\sum_{j=1}^{n} q_{i j} k_{j}\left(y_{j}(t)\right) \\
+J_{i},
\end{array}\right.
$$

where $a_{i j}, b_{i j}, p_{i j}$, and $q_{i j}$ represents the synaptic strength of the $i$ th neuron against the $j$ th neuron at time $t$ which are continuous and bounded, $c_{i},-d_{i}$ are constants, and $f_{j}(*), g_{j}(*), p_{j}(*)$, and $q_{j}(*)$ are nonlinear activation functions.

Suppose system (3) has a unique solution $(u, v)$ with any initial values of $t_{0}$ and $x_{0}$, here $u(t)=u\left(t, t_{0}, x_{0}\right), v(t)=$ $v\left(t, t_{0}, x_{0}\right)$ and there is a trivial solution $(0,0)$.

According to the above description, this paper gives a DAS model with deviation variable function:

$$
\left\{\begin{array}{l}
\frac{\mathrm{d} x_{i}(t)}{\mathrm{d} t}=-c_{i} x_{i}(t)+\sum_{j=1}^{n} a_{i j} f_{j}\left(x_{j}(\beta(t))+\sum_{j=1}^{n} b_{i j} g_{j}\left(y_{j}(t)\right)\right. \\
+I_{i} \\
0=-d_{i} y_{i}(t)+\sum_{j=1}^{n} p_{i j} h_{j}\left(x_{j}(t)\right)+\sum_{j=1}^{n} q_{i j} k_{j}\left(y_{j}(t)\right) \\
+J_{i},
\end{array}\right.
$$

where $t \geq t_{0} \geq 0, a_{i j}$ represents the synaptic strength of the $i$ th neuron against the $j$ th neuron at time $\beta(t)$, $b_{i j}, p_{i j}$, and $q_{i j}$ also expressed as synaptic strength. $c_{i},-d_{i}$ are constants, and $f_{j}(*), g_{j}(*), p_{j}(*)$, and $q_{j}(*)$ are nonlinear activation functions. Here (3) can be viewed as an undisturbed model of (4).

Next, DAS with deviation functions and stochastic disturbed are introduced.

$$
\left\{\begin{array}{l}
\mathrm{d} x_{i}(t)=\left[-c_{i} x_{i}(t)+\sum_{j=1}^{n} a_{i j} f_{j}\left(x_{j}(\beta(t))+\sum_{j=1}^{n} b_{i j} g_{j}\left(y_{j}(t)\right)\right.\right. \\
\left.+I_{i}\right] \mathrm{d} t+\sigma x_{i}(t) \mathrm{d} W(t) \\
0=-d_{i} y_{i}(t)+\sum_{j=1}^{n} p_{i j} h_{j}\left(x_{j}(t)\right)+\sum_{j=1}^{n} q_{i j} k_{j}\left(y_{j}(t)\right) \\
+J_{i} .
\end{array}\right.
$$

The deviation functions $\beta(t)=\eta_{k}, \alpha_{k}<\eta_{k}<\alpha_{k+1}$, $t \in\left[\alpha_{k}, \alpha_{k+1}\right), k \in N, f, g: R^{+} \times R^{n} \longrightarrow R^{n}$ and $\sigma$ represent noise intensity.

Remark 1. $\beta(t)$ enables the system to have both advanced and deferred characteristics. System (4) is advanced when $\alpha_{k} \leq t<\eta_{k}$; hence, $\eta_{k}<t<\alpha_{k+1}$, (4) is deferred. The DASs can be approximately regarded as a mixed system because of the strong dependence of the algebraic variables on the differential variables.

2.3. Preliminaries. The following eight hypotheses are presented:

$\left(A_{1}\right)$

$$
\begin{gathered}
\left|f_{j}\left(x_{1}\right)-f_{j}\left(x_{2}\right)\right| \leq L_{j}^{1}\left|x_{1}-x_{2}\right|, \\
\left|g_{j}\left(y_{1}\right)-g_{j}\left(y_{2}\right)\right| \leq L_{j}^{2}\left|y_{1}-y_{2}\right|, \\
\left|h_{j}\left(x_{1}\right)-h_{j}\left(x_{2}\right)\right| \leq L_{j}^{3}\left|x_{1}-x_{2}\right|, \\
\left|k_{j}\left(y_{1}\right)-k_{j}\left(y_{2}\right)\right| \leq L_{j}^{4}\left|y_{1}-y_{2}\right|,
\end{gathered}
$$

where $\quad x_{1}, x_{2} \in R^{n}, y_{1}, y_{2} \in R^{n}, \quad L_{j}^{1}>0, L_{j}^{2}>0, L_{j}^{3}>$ $0, L_{j}^{4}>0$ and $j=1, \ldots, n$. It is worth noting that $f_{j}(*), g_{j}(*), p_{j}(*)$, and $q_{j}(*)$ are satisfied local Lipschitz conditions.

$\left(A_{2}\right)$ For any $j=1,2, \ldots, n, f_{j}, g_{j}, h_{j}, k_{j}(0)=0$.

$\left(A_{3}\right)$ There exists a constant $\alpha>0$, satisfied $\alpha_{k+1}-\alpha_{k} \leq \alpha, k \in N$.

$$
\begin{aligned}
& \left(A_{4}\right) m_{1}=\max _{1 \leq i \leq n}\left\{c_{i}+\rho L_{i}^{2} \sum_{j=1}^{n}\left|b_{j i}\right|\right\} . \\
& \left(A_{5}\right) m_{2}=\max _{1 \leq i \leq n}\left\{L_{i}^{1} \sum_{j=1}^{n}\left|a_{j i}\right|\right\} . \\
& \left(A_{6}\right) \alpha\left[m_{2}-m_{1}\left(1+\alpha m_{2}\right) \exp \left\{\alpha m_{1}\right\}\right]<1 . \\
& \left(A_{7}\right) 16 m_{2}^{2} \alpha+\alpha\left(8 m_{1}^{2}+4 m_{3}^{2}\right) \\
& \left(2+16 m_{2}^{2} \alpha\right) \exp \left\{8 m_{1}^{2}+4 \sigma^{2}\right\}<1 .
\end{aligned}
$$

$$
\left(A_{8}\right) \rho=\frac{L_{i}^{3} \sum_{j=1}^{n}\left|p_{j i}\right|}{\left|d_{i}\right|-L_{i}^{4} \sum_{j=1}^{n}\left|q_{j i}\right|} .
$$

Remark 2. It is noticing that the DASs studied in this paper are all index-1, if and only if

$$
\left|d_{i}\right|-\sum_{j=1}^{n}\left|q_{i j} k_{j}^{\prime}\left(y_{j}(t)\right)\right|>0 .
$$

Based on the existence and uniqueness theorem of solutions of differential equations and existing research results, if $\left(A_{1}\right)$ and $\left(A_{2}\right)$ hold, the index-1 DASs in this research exist unique solutions $(x(t), y(t))$, which are from any initial value $\left(x_{0}, y_{0}\right)$.

\subsection{Properties}

Definition 1. For any $\left(t_{0}, x_{0}\right) \in R^{+} \times R^{n}$, there exist constants $l>0, \varsigma>0$, such that 


$$
\left\|x\left(t ; t_{0}, x_{0}\right)\right\| \leq l\left\|x_{0}\right\| \exp \left\{-\varsigma\left(t-t_{0}\right)\right\}, t \geq t_{0} \geq 0 .
$$

Then, system (4) is globally exponentially stable.

Definition 2. System (5) is almost surely exponentially stable, if for any $t_{0} \in R^{+}, y_{0} \in R^{n}$, there exist $\Delta>0, \Lambda>0$, such that

$$
\|u(t)\| \leq \Delta\left\|y_{0}\right\| \exp \left\{-\Lambda\left(t-t_{0}\right)\right\}, \quad t \geq t_{0} \geq 0,
$$

holds a.e.

Definition 3. For any $t_{0} \in R^{+}, y_{0} \in R^{n}$, system (5) satisfies the mean-square exponentially stable; then, there exist $\Delta>0, \Lambda>0$, such that

$$
E\left\|x\left(t ; t_{0}, y_{0}\right)\right\|^{2} \leq \Delta\left\|y_{0}\right\| \exp \left\{-\Lambda\left(t-t_{0}\right)\right\}, \quad t \geq t_{0} \geq 0 .
$$

\section{Main Results}

The following lemma gives the relationship between the state $x(t)$ and the deviation function $\beta(t)$ in system (4).

Lemma 1. Let $\left(A_{1}\right)-\left(A_{8}\right)$ hold, $(\vec{x}(t), \vec{y}(t))$ is the solution of system (4), and then

$$
\|\vec{x}(\beta(t))\| \leq \lambda\|\vec{x}(t)\|
$$

holds for $t \in R^{+}$, where

$$
\lambda=\left[1-\alpha m_{2}-\alpha m_{1}\left(1+\alpha m_{2}\right) \exp \left\{\alpha m_{1}\right\}\right]^{-1} .
$$

Proof. Fix $t \in N$, for any $t \in\left[\alpha_{k}, \alpha_{k+1}\right)$, for dynamic equation, we obtain

$$
\begin{aligned}
& \|\vec{x}(t)\| \\
& \leq\left\|\vec{x}\left(\eta_{k}\right)\right\|+\sum_{i=1}^{n}\left\{\int_{\eta_{k}}^{t} \mid\left[a_{i} \vec{x}_{i}(s)+\sum_{i=1}^{n} b_{i j} f_{j}\left(\vec{x}_{j}(\beta(t))\right)\right.\right. \\
& \left.\left.\quad+\sum_{i=1}^{n} c_{i j} f_{j}\left(\vec{y}_{j}(s)\right)\right] \mid \mathrm{d} s\right\} \\
& \leq \| \vec{x}\left(\eta_{k}\right) \mid+\sum_{i=1}^{n}\left\{\int_{\eta_{k}}^{t} \mid\left[a_{i}\left|\vec{x}_{i}(s)\right|+\sum_{i=1}^{n} b_{i j} L_{j}^{1} \mid \vec{x}_{j}(\beta(t) \mid\right.\right. \\
& \left.\left.\quad+\sum_{i=1}^{n} c_{i j} L_{j}^{2}\left|\vec{y}_{j}(s)\right|\right] \mid \mathrm{d} s\right\} .
\end{aligned}
$$

For static equation,

\section{$\|\vec{y}(t)\|$}

$$
\begin{aligned}
& =\frac{1}{d_{i}} \sum_{i=1}^{n}\left|\left(\sum_{i=1}^{n} p_{i j} h_{j}\left(\vec{x}_{j}(t)\right)+\sum_{i=1}^{n} q_{i j} k_{j}\left(\vec{y}_{j}(t)\right)\right)\right| \\
& \leq \frac{1}{d_{i}}\left(L_{i}^{3} \sum_{i=1}^{n}\left|p_{i j}\right| \sum_{i=1}^{n}\left|\vec{x}_{i}(t)\right|+L_{i}^{4} \sum_{i=1}^{n}\left|q_{i j}\right| \sum_{i=1}^{n}\left|\vec{y}_{i}(t)\right|\right) \\
& =\frac{1}{d_{i}}\left(L_{i}^{3} \sum_{i=1}^{n}\left|p_{i j}\right|\left\|\vec{x}_{i}(t)\right\|+L_{i}^{4} \sum_{i=1}^{n}\left|q_{i j}\right|\left\|\left|\vec{y}_{i}(t)\right|\right\|\right),
\end{aligned}
$$

then

$$
\begin{aligned}
\|\vec{y}(t)\| & \leq \frac{L_{i}^{3} \sum_{i=1}^{n}\left|p_{i j}\right|}{\left|d_{i}\right|-L_{i}^{4} \sum_{i=1}^{n}\left|q_{i j}\right|}\|\vec{x}(t)\| \\
& =\rho\left|\left\|\vec{x}_{j}(t)\right\|\right|,
\end{aligned}
$$

where

$$
\rho=\frac{L_{i}^{3} \sum_{i=1}^{n}\left|p_{i j}\right|}{\left|d_{i}\right|-L_{i}^{4} L_{i}^{4} \sum_{i=1}^{n}\left|q_{i j}\right|} .
$$

Combining (14) with (16), we obtain

$$
\begin{aligned}
\|\vec{x}(t)\| \leq & \left\|\vec{x}\left(\eta_{k}\right)\right\|+\sum_{i=1}^{n}\left\{\int _ { \eta _ { k } } ^ { t } \left[c_{i}\left|\vec{x}_{i}(s)\right|\right.\right. \\
& \left.\left.+\sum_{j=1}^{n} a_{i j} L_{j}^{1}\left|\vec{x}_{j}\left(\eta_{k}(s)\right)\right|+\rho \sum_{j=1}^{n} b_{i j} L_{j}^{2}\left|\vec{x}_{j}(s)\right|\right] \mathrm{d} s\right\} \\
\leq & \left\|\vec{x}\left(\eta_{k}\right)\right\|+\left\{\int _ { \eta _ { k } } ^ { t } \left[\sum_{i=1}^{n}\left(c_{i}+\rho \sum_{j=1}^{n} b_{j i} L_{i}^{2}\right)\left|\vec{x}_{i}(s)\right|\right.\right. \\
& \left.\left.\left.+\sum_{i=1}^{n} \sum_{j=1}^{n}\right] a_{i j} L_{i}^{1}\left|\vec{x}_{i}\left(\eta_{k}(s)\right)\right|\right] \mathrm{d} s\right\} \\
\leq & \left(1+m_{2} \alpha\right)\left\|\vec{x}\left(\eta_{k}\right)\right\|+\int_{\eta_{k}}^{t} m_{1}\|\vec{x}(s)\| \mathrm{d} s .
\end{aligned}
$$

According to the Gronwall-Bellman formula, we can get

$$
\|\vec{x}(t)\| \leq\left(1+\alpha m_{2}\right) \exp \alpha m_{1}\left\|\vec{x}\left(\eta_{k}\right)\right\| .
$$

In the same way, for $t \in\left[\alpha_{k}, \alpha_{k+1}\right)$, we obtain

$$
\begin{aligned}
& \left\|\vec{x}\left(\eta_{k}\right)\right\| \leq\|\vec{x}(t)\|+\sum_{i=1}^{n}\left\{\int _ { \eta _ { k } } ^ { t } \left[c_{i}\left|\vec{x}_{i}(s)\right|+\sum_{j=1}^{n} a_{i j} L_{j}^{1}\right.\right. \\
& \left.\left.\left|\vec{x}_{j}\left(\eta_{k}(s)\right)\right|+\rho \sum_{j=1}^{n} b_{j i} L_{j}^{2}\left|\vec{x}_{j}(s)\right|\right] \mathrm{d} s\right\} \\
& \leq\|\vec{x}(t)\|+\alpha m_{2}\left\|\vec{x}\left(\eta_{k}\right)\right\|+\int_{\eta_{k}}^{t} m_{1}\|\vec{x}(s)\| \mathrm{d} s .
\end{aligned}
$$


Then, we have

$$
\begin{aligned}
\left\|\vec{x}\left(\eta_{k}\right)\right\| \leq & \|\vec{x}(t)\|+\alpha m_{2}\left\|\vec{x}\left(\eta_{k}\right)\right\| \\
& +\alpha m_{1}\left(1+\alpha m_{2}\right) \exp \left\{\alpha m_{1}\right\}\left\|\vec{x}\left(\eta_{k}\right)\right\|,
\end{aligned}
$$

together with (19) and (21),

$$
\begin{aligned}
\left\|\vec{x}\left(\eta_{k}\right)\right\| & \leq\left[\left[1-\alpha m_{2}-\alpha m_{1}\left(1+\alpha m_{2}\right) \exp \left\{\alpha m_{1}\right\}\right]^{-1}\|\vec{x}(t)\|\right] \\
& =\lambda\|\vec{x}(t)\|,
\end{aligned}
$$

where $\lambda=\left[1-\alpha m_{2}-\alpha m_{1}\left(1+\alpha m_{2}\right) \exp \left\{\alpha m_{1}\right\}\right]^{-1}$.

For any $t \in\left[\alpha_{k}, \alpha_{k+1}\right)$, because of the arbitrariness of $t$ and $k,(9)$ is valid for all $t \in R^{+}$.

Lemma 2. Let $\left(A_{1}\right)-\left(A_{8}\right)$ hold, $(\mathscr{X}(t), \mathscr{Y}(t))$ is the solution of system (5), and then

$$
E\|\mathscr{X}(\beta(t))\|^{2} \leq \omega E\|\mathscr{X}(t)\|^{2},
$$

holds for $t \in R^{+}$, where $\omega=2(1-\omega)^{-1}$, and

$$
\omega=16 m_{2}^{2} \alpha+\alpha\left(8 m_{1}^{2}+4 m_{3}^{2}\right)\left(2+16 m_{2}^{2} \alpha\right) \exp \left\{8 m_{1}^{2}+4 \delta^{2}\right\} .
$$

Proof. For any $t \in R^{+}$, there exists $k \in N$, such that $t \in\left[\alpha_{k}, \alpha_{k+1}\right), \beta(t)=\eta_{k}$, and then

$$
\begin{aligned}
E\|\mathscr{X}(t)\|^{2} \leq & 2 E\left\|\mathscr{X}\left(\eta_{k}\right)\right\|^{2}+2 E \mid \sum_{i=1}^{n}\left\{\int _ { \eta _ { k } } ^ { t } \left[c_{i}\left|\mathscr{X}_{i}(s)\right|\right.\right. \\
& +L_{j}^{1} \sum_{j=1}^{n} a_{i j}\left|\mathscr{X}\left(\eta_{k}\right)\right| \\
& \left.\left.+\rho L_{j}^{2} b_{i j}\left|\mathscr{X}_{j}(s)\right|\right] \mathrm{d} s+\sigma \int_{\eta_{k}}^{t}\left|\mathscr{X}_{j}(s)\right| \mathrm{d} W(s)\right\}\left.\right|^{2} \\
\leq & 2 E\left\|\mathscr{X}\left(\eta_{k}\right)\right\|^{2}+8 m_{1} \int_{\eta_{k}}^{t} E\|\mathscr{X}(s)\|^{2} \mathrm{~d} s \\
& +8 m_{2} \alpha^{2} E\left\|\mathscr{X}\left(\eta_{k}\right)\right\|^{2}+4 \sigma^{2} \int_{\eta_{k}}^{t} E\|\mathscr{X}(s)\|^{2} \mathrm{~d} s \\
= & \left(2+8 m_{2} \alpha^{2}\right) E\left\|\mathscr{X}\left(\eta_{k}\right)\right\|^{2} \\
& +\left(8 m_{1}+4 \sigma^{2}\right) \int_{\eta_{k}}^{t} E\|\mathscr{X}(s)\|^{2} \mathrm{~d} s .
\end{aligned}
$$

According to the Gronwall-Bellman formula to (25), we can get

$$
E\|\mathscr{X}(t)\|^{2} \leq\left(2+8 m_{2} \alpha^{2}\right) E\left\|\mathscr{X}\left(\eta_{k}\right)\right\|^{2} \exp \left\{8 m_{1}+4 m_{2}^{2}\right\} .
$$
have

In the same way, from above, for any $t \in\left[\alpha_{k}, \alpha_{k+1}\right)$, we

$$
\begin{aligned}
E\left\|\mathscr{X}\left(\eta_{k}\right)\right\|^{2} & \\
\leq & 2 E\|\mathscr{X}(t)\|^{2}+8 m_{1}^{2} \int_{\eta_{k}}^{t} E\|\mathscr{X}(s)\|^{2} \mathrm{~d} s \\
& +16 m_{2}^{2} \alpha^{2} E\left\|\mathscr{X}\left(\eta_{k}\right)\right\|^{2}+4 \sigma^{2} \int_{\eta_{\partial}}^{t} E\|\mathscr{X} x(s)\|^{2} \mathrm{~d} s \\
& 2 E\|\mathscr{X}(t)\|^{2}+16 m_{2}^{2} \alpha^{2} E\left\|\mathscr{X}\left(\eta_{k}\right)\right\|^{2} \\
& +\left(8 m_{1}^{2}+4 \sigma^{2}\right) \int_{\eta_{k}}^{t} E\|\mathscr{X}(s)\|^{2} \mathrm{~d} s \\
\leq & 2 E\|\mathscr{X}(t)\|^{2}+16 m_{2}^{2} \alpha^{2} E\left\|\mathscr{X}\left(\eta_{k}\right)\right\|^{2}+\alpha\left(8 m_{1}^{2}+4 \sigma^{2}\right) \\
& \left(2+16 m_{2}^{2} \alpha\right) \exp \left\{8 m_{1}^{2}+4 \sigma^{2}\right\} E\left\|\mathscr{X}\left(\eta_{k}\right)\right\|^{2} \\
= & 2 E\|\mathscr{X}(t)\|^{2}+\omega E\left\|\mathscr{X}\left(\eta_{k}\right)\right\|^{2},
\end{aligned}
$$

where

$$
\omega=16 m_{2}^{2} \alpha^{2}+\alpha\left(8 m_{1}^{2}+4 \sigma^{2}\right)\left(2+16 m_{2}^{2} \alpha\right) \exp \left\{8 m_{1}^{2}+4 \sigma^{2}\right\} .
$$

From (27), it follows

$$
E\left\|\mathscr{X}\left(\eta_{k}\right)\right\|^{2} \leq 2(1-\omega)^{-1} E\|\mathscr{X}(t)\|^{2}=\varpi E\|\mathscr{X}(t)\|^{2},
$$

where $₫=2(1-\omega)^{-1}$.

3.1. System Stability. Next, the robustness of the deviation term to the global exponential stability of system (4) is discussed.

Theorem 1. Suppose that the conditions $\left(A_{1}\right)-\left(A_{8}\right)$ hold and system (3) is globally exponentially stable, if $\alpha<\min \left(\eta / 2, \mathscr{I}^{1}, \mathscr{J}^{2}\right)$, where $\mathscr{I}^{1}$ is the only positive solution $\hat{x}$ to the transcendental equation

$$
\begin{aligned}
& l \exp \{-\varsigma(\eta-\hat{x})\}+\frac{m_{2}(1+\lambda) l}{\varsigma} \\
& \quad \times \exp \left\{2 \eta \left(m_{1}+2 m_{2}+\left[1-\hat{x} m_{2}-\hat{x} m_{1}\right.\right.\right. \\
& \left.\left.\left.\left(1+\hat{x} m_{2}\right) \exp \left\{\hat{x} m_{1}\right\}\right]^{-1} m_{2}\right)\right\}=1
\end{aligned}
$$

and $\mathscr{I}^{2}$ is the only positive solution $\check{x}$ to this transcendental equation

$$
\breve{x}\left[m_{2}+m_{1}\left(1+\breve{x} m_{2}\right) \exp \left\{\breve{x} m_{1}\right\}\right]=1,
$$

where $\eta>\ln (l) / \varsigma>0$.

Proof. $(\vec{x}(t), \vec{y}(t))$ is the solution to (4), and $(u(x), v(x))$ is the solution to system (3), combined with lemma (3), for any $t \geq t_{0}>0$, for dynamic equation 


$$
\begin{aligned}
\|u(t)-\vec{x}(t)\|= & \sum_{i=1}^{n} \int_{t_{0}}^{t}\left[a_{i}\left|u_{i}(s)-\vec{x}_{i}(s)\right|+\sum_{i=1}^{n} \mid b_{i j} \| f_{j}\left(u_{j}(s)\right)\right. \\
& -f_{j}\left(\vec{x}_{j}\left(\eta_{k}\right)\right) \mid \\
& \left.+\sum_{i=1}^{n}\left|c_{i j}\right|\left|g_{j}\left(v_{j}(s)-g_{j}\left(\vec{y}_{j}(s)\right)\right)\right|\right] \mathrm{d} s \\
\leq & \sum_{i=1}^{n} \int_{t_{0}}^{t}\left[a_{i}\left|u_{i}(s)+\vec{x}_{i}(s)\right|\right. \\
& +L_{j}^{1} \sum_{i=1}^{n}\left|b_{i j} \|\right| u_{j}(s)-\vec{x}_{j}\left(\eta_{k}\right) \mid \\
& \left.+L_{j}^{2} \sum_{i=1}^{n}\left|c_{i j} \| v_{j}(s)-\vec{y}_{j}(s)\right|\right] \mathrm{d} s .
\end{aligned}
$$

For static equation,

$$
\begin{aligned}
\| v(t) & -\vec{y}(t) \| \\
= & \frac{1}{d_{i}} \sum_{i=1}^{n}\left(L_{j}^{3} \sum_{j=1}^{n}\left|p_{i j} \| u_{j}(t)-\vec{x}_{j}(t)\right|\right. \\
& \left.+L_{j}^{4} \sum_{j=1}^{n}\left|q_{i j} \| v_{j}(t)-\vec{y}_{j}(t)\right|\right),
\end{aligned}
$$

then

$$
\begin{aligned}
& \|v(t)-\vec{y}(t)\| \\
& \quad \leq \frac{L_{i}^{3} \sum_{j=1}^{n}\left|p_{j i}\right|}{\left|d_{i}\right|-L_{i}^{4} \sum j=1^{n}\left|q_{j i}\right|}\|u(t)-\vec{x}(t)\| \\
& \quad=\rho\|u(t)-\vec{x}(t)\| .
\end{aligned}
$$

The following can be obtained from (32) and (34):

$$
\begin{aligned}
\|u(t)-\vec{x}(t)\| \leq & \sum_{i=1}^{n} \int_{t_{0}}^{t}\left[a_{i}\left|u_{i}(s)-\vec{x}_{i}(s)\right|\right. \\
& +L_{j}^{1} \sum_{j=1}^{n}\left|b_{i j} \| u_{j}(s)-\vec{x}_{j}\left(\eta_{k}\right)\right| \\
& \left.+\rho L_{j}^{2} \sum_{j=1}^{n}\left|b_{i j} \| u_{j}(s)-\vec{x}_{j}(s)\right|\right] \mathrm{d} s \\
\leq & m_{1} \int_{t_{0}}^{t}\|u(s)-\vec{x}(s)\| \mathrm{d} s \\
& +m_{2} \int_{t_{0}}^{t}\left\|u(s)-\vec{x}\left(\eta_{k}\right)\right\| \mathrm{d} s \\
\leq & \left(m_{1}+m_{2}\right) \int_{t_{0}}^{t}\|u(s)-\vec{x}(s)\| \mathrm{d} s \\
& +m_{2} \int_{t_{0}}^{t}\left\|\vec{x}(s)-\vec{x}\left(\eta_{k}\right)\right\| \mathrm{d} s \\
\leq & \left(m_{1}+m_{2}\right) \int_{t_{0}}^{t}\|u(s)-\vec{x}(s)\| \mathrm{d} s \\
& +m_{2} \int_{t_{0}}^{t}\left(\|\vec{x}(s)\|+\left\|\vec{x}\left(\eta_{k}\right)\right\|\right) \mathrm{d} s \\
\leq & \left(m_{1}+m_{2}\right) \int_{t_{0}}^{t}\|u(s)-\vec{x}(s)\| \mathrm{d} s \\
& +m_{2}(1+\lambda) \int_{t_{0}}^{t}\|\vec{x}(s)\| \mathrm{d} s \\
\leq & \left(m_{1}+2 m_{2}+\lambda m_{2}\right) \int_{t_{0}}^{t}\|u(s)-\vec{x}(s)\| \mathrm{d} s \\
& +m_{2}(1+\lambda) \int_{t_{0}}^{t}\|u(s)\| \mathrm{d} s \\
& +x(s)
\end{aligned}
$$

Due to the characteristics of system (3), from (35), when $t \leq t_{0} \leq 0$, then

$$
\begin{aligned}
\|u(t)-\vec{x}(t)\| \leq & \left(m_{1}+2 m_{2}+\lambda m_{2}\right) \int_{t_{0}}^{t}\|u(s)-\vec{x}(s)\| \mathrm{d} s \\
& +m_{2}(1+\lambda) \int_{t_{0}}^{t} l\left\|\vec{x}_{0}\right\| \exp \left\{-k\left(s-t_{0}\right)\right\} \mathrm{d} s \\
\leq & \left(m_{1}+2 m_{2}+\lambda m_{2}\right) \int_{t_{0}}^{t}\|u(s)-\vec{x}(s)\| \mathrm{d} s \\
& +\frac{m_{2}(1+\lambda) l}{k}\left\|\vec{x}_{0}\right\| .
\end{aligned}
$$

By the Gronwall-Bellman inequality to (36), for any $t_{0}+\eta \leq t \leq t_{0}+2 \eta$, we have

$$
\|u(t)-\vec{x}(t)\| \leq \frac{m_{2}(1+\lambda) l}{k}\left\|\vec{x}_{0}\right\| \exp \left\{2 \eta\left(m_{1}+2 m_{2}+\lambda m_{2}\right)\right\} .
$$

Then

$$
\begin{aligned}
\|\vec{x}(t)\| \leq & \|u(t)\|+\|u(t)-\vec{x}(t)\| \\
& \leq\|u(t)\| \\
& \quad+\frac{m_{2}(1+\lambda) l}{k}\left\|\vec{x}_{0}\right\| \exp \left\{2 \eta\left(m_{1}+2 m_{2}+\lambda m_{2}\right)\right\} .
\end{aligned}
$$

For any $t_{0}-\alpha+\eta \leq t \leq t_{0}-\alpha+2 \eta$, by (23), then $\eta-\alpha \leq t-t_{0} \leq 2 \eta-\alpha$, so

$\|\vec{x}(t)\| \leq l\left\|\vec{x}\left(t_{0}\right)\right\| \exp \{-k(\eta-\alpha)\}$

$$
\begin{aligned}
& +\frac{m_{2}(1+\lambda) l}{k}\left\|\vec{x}_{0}\right\| \exp \left\{2 \eta\left(m_{1}+2 m_{2}+\lambda m_{2}\right)\right\} \\
\triangleq & \delta\left\|\vec{x}_{0}\right\|,
\end{aligned}
$$

where

$$
\begin{aligned}
& \delta=l \exp \{-k(\eta-\alpha)\}+\frac{m_{2}(1+\lambda) l}{k} \\
& \exp \left\{2 \eta\left(m_{1}+2 m_{2}+\lambda m_{2}\right)\right\} .
\end{aligned}
$$

Fix $\gamma=-\ln (\delta) / \eta$, then

$$
\|\vec{x}(t)\| \leq \exp \{-\eta \gamma\}\left\|\vec{x}_{0}\right\|
$$

System (4) has a unique solution that satisfies the following equation:

$$
\begin{aligned}
\vec{x} & \left(t ; t_{0}, y_{0}\right) \\
& =\vec{x}\left(t ; t_{0}+(m-1) \eta, \vec{x}\left(t_{0}+(m-1) \eta ; t_{0}, x_{0}\right)\right),
\end{aligned}
$$

where $m$ is a positive integer; therefore, combining (41) and (26), for all $t \leq t_{0}-\alpha+m \eta$, we have 


$$
\begin{aligned}
& \| \vec{x} \\
& \quad=\vec{x}\left(t ; t_{0}, x_{0}\right) \| \\
& \quad \leq \exp -\{\eta \gamma\} \| \vec{x}\left(t_{0}+(m-1) \eta ; t_{0}, \vec{x}_{0} \|\right. \\
& \quad=\exp \{-\eta \gamma\} \| \vec{x}\left(t_{0}+(m-2) \eta, \vec{x}\left(t_{0}+(m-2)\right) \eta ; t_{0}, \vec{x}_{0}\right) \\
& \quad \leq \exp \{-m \eta \gamma\}\left\|\vec{x}_{0}\right\| .
\end{aligned}
$$

From what has been discussed above, when $t_{0}-\alpha+(m-1) \eta \leq t \leq t_{0}-\alpha+m \eta$, we have

$$
\begin{aligned}
& \left\|\vec{x}\left(t ; t_{0}, x_{0}\right)\right\| \\
& \quad \leq \exp \left\{-\gamma\left(t-t_{0}\right)\right\} \exp \{\gamma(\eta-\alpha)\}\left\|\vec{x}_{0}\right\|,
\end{aligned}
$$

when $t_{0} \leq t \leq t_{0}-\alpha+\eta$, the above equation is also true, so system (4) achieved global exponential stability, which is verified.

Remark 3. Theorem 1 shows that the system remains globally asymptotically stable when the length of the interval of the deviation function $\beta(t)$ of the disturbed system is less than the lower bound, that is, $\min \left(\eta / 2, \mathscr{J}^{1}, \mathscr{J}^{2}\right)$.

Theorem 2. Assuming that $\left(A_{1}\right)-\left(A_{8}\right)$ hold and $(\mathscr{X}(t), \mathscr{Y}(t))$ is the solution of system (3) from $\left(X_{0}, \mathscr{Y}_{0}\right)$ and system (3) is globally exponentially stable, then system (5) is mean-square exponentially stable when $|\sigma|<\bar{\sigma} /$ $\sqrt{6}, \alpha<\min \left(o / 2, \mathcal{I}^{1}\right)$, where $\bar{\sigma}$ is the only positive solution $\hat{y}$ of equations as follows:

$$
\begin{aligned}
& 2 l \exp \{-k o\}+2\left[288 m_{2}^{2} o+6 \hat{y}^{2}\right] \frac{2 o l}{k} \\
& \quad \times \exp \left\{\left[12 o\left(m_{1}^{2}+2 m_{2}^{2}\right)+288 m_{2}^{2} o+6 \hat{y}^{2}\right]\right\}=1 .
\end{aligned}
$$

$\alpha$ is the only positive solution $\check{y}$ of the transcendental equation:

$$
\begin{aligned}
& 2 l \exp \{-k(o-\breve{y})\}+2\left[96 m_{2}^{2} o(1+\overline{\bar{\omega}})+\bar{\sigma}^{2}\right] \frac{o l}{k} \\
& \quad \times \exp \left\{\left[12 o\left(m_{1}^{2}+2 m_{2}^{2}\right)+288 m_{2}^{2} o+6 \hat{y}^{2}\right]\right\}=1,
\end{aligned}
$$

where $o>\ln (l) / \varsigma>0$,

$$
\bar{\omega}=16 m_{2}^{2} \breve{y}+\breve{y}\left(8 m_{1}^{2}+4 \bar{\sigma}^{2}\right)\left(2+16 m_{2}^{2} \breve{y}\right) \exp \left\{8 m_{1}^{2}+4 \bar{\sigma}^{2}\right\} \text {. }
$$

Proof. Fix $\mathscr{X}\left(t ; t_{0}, \mathscr{X}_{0}\right)=\mathscr{X}(t), u\left(t ; t_{0}, \mathscr{X}_{0}\right)=u(t)$, for any $t \geq t_{0}>0$, and we have

$$
\begin{aligned}
& E\|\mathscr{X}(t)-u(t)\| \\
& =E \mid \sum_{i=1}^{n} \int_{t_{0}}^{t}\left[c_{i}\left|\mathscr{X}_{i}(s)-u_{i}(s)\right|+\sum_{i=1}^{n} a_{i j} \mid f_{j}\left(\mathscr{X}_{j}(\beta(s))\right)\right. \\
& \left.-f_{j}\left(u_{j}(s)\right)\left|+\sum_{i=1}^{n} b_{i j}\right| g_{j}\left(y_{i}(s)\right)-g_{j}\left(v_{j}(s)\right) \mid\right] \mathrm{d} s \\
& +\left.\int_{t_{0}}^{t} \sigma X_{i}(s) \mathrm{d} W(s)\right|^{2} \\
& \leq E \mid \int_{t_{0}}^{t} m_{1}\|\mathscr{X}(s)-u(s)\| \mathrm{d} s+\int_{t_{0}}^{t} m_{2}\left\|\mathscr{X}\left(\eta_{k}\right)-u(s)\right\| \\
& +\left.\sigma \int_{t_{0}}^{t}\|\mathscr{X}(s)\| \mathrm{d} W(s)\right|^{2} \\
& \leq 6\left(t-t_{0}\right) m_{1}^{2} \int_{t_{0}}^{t} E\|\mathscr{X}(s)-u(s)\|^{2} \mathrm{~d} s+6\left(t-t_{0}\right) m_{2}^{2} \\
& \int_{t_{0}}^{t} E\left\|\mathscr{X}\left(\eta_{k}\right)-u(s)\right\|^{2} \mathrm{~d} s+3 \sigma^{2} \int_{t_{0}}^{t} E\|\mathscr{X}(s)\|^{2} \mathrm{~d} s \\
& \leq 6\left(t-t_{0}\right) m_{1}^{2} \int_{t_{0}}^{t} E\|\mathscr{X}(s)-u(s)\|^{2} \mathrm{~d} s+12\left(t-t_{0}\right) m_{2}^{2} \\
& \int_{t_{0}}^{t} E\|\mathscr{X}(s)-u(s)\|^{2} \mathrm{~d} s+24\left(t-t_{0}\right) m_{2}^{2} \int_{t_{0}}^{t} E\|\mathscr{X}(s)\|^{2} \mathrm{~d} s \\
& 24\left(t-t_{0}\right) m_{2}^{2} \int_{t_{0}}^{t} E\left\|\mathscr{X}\left(\eta_{k}\right)\right\|^{2} \mathrm{~d} s+3 m_{3}^{2} \int_{t_{0}}^{t} E\|\mathscr{X}(s)\|^{2} \mathrm{~d} s \\
& =6\left(t-t_{0}\right)\left(m_{1}^{2}+2 m_{2}^{2}\right) \int_{t_{0}}^{t} E\|\mathscr{X}(s)-u(s)\|^{2} \mathrm{~d} s \\
& +\left[24\left(t-t_{0} m_{2}^{2}+\sigma^{2}\right)\right] \times \int_{t_{0}}^{t} E\|\mathscr{X}(s)\|^{2} \mathrm{~d} s \\
& +24\left(t-t_{0}\right) m_{2}^{2} \int_{t_{0}}^{t} E\left\|\mathscr{X}\left(\eta_{k}\right)\right\|^{2} \mathrm{~d} s \\
& \leq 6\left(t-t_{0}\right)\left(m_{1}^{2}+2 m_{2}^{2}\right) \int_{t_{0}}^{t} E\|\mathscr{X}(s)-u(s)\|^{2} \mathrm{~d} s \\
& +\left[24 m_{2}^{2}\left(t-t_{0}\right)(1+\varpi)+3 \sigma^{2}\right] \int_{t_{0}}^{t} E\|\mathscr{X}(s)\|^{2} \mathrm{~d} s \\
& {\left[6\left(t-t_{0}\right)\left(m_{1}^{2}+2 m_{2}^{2}\right)+48 m_{2}^{2}\left(t-t_{0}\right)(1+\varpi)+6 \sigma^{2}\right]} \\
& \int_{t_{0}}^{t} E\|\mathscr{X}(s)-u(s)\|^{2} \mathrm{~d} s+\left[48 m_{2}^{2}\left(t-t_{0}\right)(1+\varpi)\right. \\
& \left.+6 \sigma^{2}\right] \frac{l}{\varsigma}\left\|\mathscr{X}\left(t_{0}\right)\right\|\left(t-t_{0}\right)
\end{aligned}
$$

when $t_{0}+\alpha \leq t \leq t_{0}+2 o$, and by (48), then 


$$
\begin{aligned}
E \| & \mathscr{X}(t)-u(t) \|^{2} \\
& \leq\left[96 m_{2}^{2} o(1+\Phi)+6 \sigma^{2}\right] \frac{2 o l}{\varsigma}\left\|\mathscr{X}_{0}\right\|^{2} \\
& \times \exp \left\{\left[12 o\left(m_{1}^{2}+2 m_{2}^{2}\right)+48 m_{2}^{2}\left(t-t_{0}\right)(1+\Phi)+6 \sigma^{2}\right]\right\},
\end{aligned}
$$

for any $t_{0}+\alpha \leq t \leq t_{0}+2 o$, and from (31), we have

$$
\begin{aligned}
& E\|\mathscr{X}(t)\|^{2} \leq 2 E\|u(t)\|^{2}+2 E\|\mathscr{X}(t)-u(t)\|^{2} \\
& \leq 2 l\left\|\mathscr{X}_{0}\right\|^{2} \exp \left\{-k\left(t-t_{0}\right)\right\}+2\left[96 m_{2}^{2} o(1+\infty)+6 \sigma^{2}\right] \\
& \frac{2 o l}{\varsigma}\left\|\bar{x}_{0}\right\|^{2} \times \exp \left\{\left[12 o\left(m_{1}^{2}+2 m_{2}^{2}\right)+96 m_{2}^{2} o(1+\infty)+6 \sigma^{2}\right]\right\} .
\end{aligned}
$$

Therefore, for any $t_{0}-\alpha+o \leq t \leq t_{0}-\alpha+2 o$,

$$
\begin{aligned}
E\|\mathscr{X}(t)\|^{2} \leq & 2 l\left\|\mathscr{X}_{0}\right\|^{2} \exp \{-k(o-\alpha)\} \\
& +2\left[96 m_{2}^{2} o(1+\Phi)+6 \sigma^{2}\right] \frac{2 o l}{\varsigma}\left\|\mathscr{X}_{0}\right\|^{2} \\
& \times \exp \left\{12 o\left(m_{1}^{2}+2 m_{2}^{2}\right)+96 m_{2}^{2} o(1+\omega)+6 \sigma^{2}\right\} \\
= & \pi\left\|\mathscr{X}_{0}\right\|^{2},
\end{aligned}
$$

where

$$
\begin{aligned}
\pi= & 2 l \exp \{-k(o-\alpha)\}+2\left[96 m_{2}^{2} o(1+\omega)+6 \sigma^{2}\right] \frac{2 o l}{\varsigma} \\
& \times \exp \left\{12 o\left(m_{1}^{2}+2 m_{2}^{2}\right)+96 m_{2}^{2} o(1+\omega)+6 \sigma^{2}\right\} .
\end{aligned}
$$

Together with (45) and (46), when $\alpha<\min \left\{o / 2, \mathscr{J}^{1}\right\},|\sigma|<\bar{\sigma} / \sqrt{6}$, we have $\pi<1$. Fix $v=-(\ln (\pi) / o)$, and then

$$
E\|\mathscr{X}(t)\|^{2} \leq \exp \{-o v\}\left\|\mathscr{X}_{0}\right\|^{2} .
$$
(5)

According to the uniqueness of the solution of system

$$
\begin{aligned}
\mathscr{X} & \left(t ; t_{0}, \mathscr{X}_{0}\right) \\
& =\mathscr{X}\left(t ; t_{0}+(m-1) o, \mathscr{X}\left(t_{0}+(m-1) o ; t_{0}, \mathscr{X}_{0}\right)\right),
\end{aligned}
$$

where $m$ is a positive integer. Combined with (53) and (54), for any $t \leq t_{0}-\alpha+m o$, we have

$$
\begin{aligned}
E \| & \mathscr{X}\left(t ; t_{0}, \mathscr{X}_{0}\right) \| \\
& =E\left\|\mathscr{X}\left(t ; t_{0}+(m-1) o, \mathscr{X}\left(t_{0}+(m-1) o ; t_{0}, \mathscr{X}_{0}\right)\right)\right\| \\
& \leq \exp \{-o v\}\left\|\mathscr{X}\left(t_{0}+(m-1) o ; t_{0}, \mathscr{X}_{0}\right)\right\|^{2} \\
& =\exp \{-o v\}\left\|\mathscr{X}\left(t_{0}+(m-2) o, \mathscr{X}\left(t_{0}+(m-2)\right) o ; t_{0}, \mathscr{X}_{0}\right)\right\|^{2} \\
& \leq \exp \{-m o v\}\left\|\mathscr{X}_{0}\right\|^{2} .
\end{aligned}
$$

So, there exists positive integer $m$, such that when $t_{0}-\alpha+(m-1) o \leq t \leq t_{0}-\alpha+m o$,

$$
E\left\|\mathscr{X}\left(t ; t_{0}, x_{0}\right)\right\|^{2} \leq \exp \left\{-v\left(t-t_{0}\right)\right\} \exp \{v(o-\alpha)\}\left\|\mathscr{X}_{0}\right\|^{2} .
$$

The proof is completed.

Remark 4. Theorem 2 shows that as long as the deviation function and noise intensity are within a certain range, system (5) is exponentially mean-square stable on the premise that system (3) is globally exponentially stable. The range, which is $|\sigma|<\bar{\sigma} / \sqrt{6}, \alpha<\min \left(o / 2, \mathscr{I}^{1}\right)$ can also be obtained by solving the transcendental equation in MATLAB.

Remark 5. Although the research in this article is based on the DASs, it is extremely dependent on the differential variable in the processing process and deals with differential variables and algebraic variables separately. This creates a situation where the index of the system eventually decreases to zero. There are even differential algebraic equations that cannot be reduced to differential equations. Therefore, a method to solve the stability problem by directly using a differential algebraic form needs to be developed.

Remark 6. Continuous Hopfield neural networks are usually used to solve optimization problems. The objective function is converted into a network energy function, and the variable of the problem corresponds to the state of the network neuron. In this way, the minimum point of the energy function is transformed into the equilibrium point of the system. When the CHNN model is used for optimization calculations, only the final state of the network evolution is needed, and there is no need to pay attention to the state evolution trajectory in the process. That is to say, as long as the step length of the time variable is long enough, there is no need to care whether the state is close to a trajectory during the numerical simulation. 


\section{Example}

In this part, two numerical examples are given to prove the validity of the theorems.

\subsection{System Description}

Example 1. Consider the following two-dimensional case of a DASs,

$$
\left\{\begin{array}{l}
\frac{\mathrm{d} x_{1}}{\mathrm{~d} t}=-x_{1}(t)-0.005 \sin ^{2}\left(x_{1}(\beta(t))\right)+0.005 \sin ^{2}\left(x_{2}(\beta(t))\right) \\
-0.005 \sin ^{2}\left(y_{1}(\beta(t))\right)+0.005 \sin ^{2}\left(y_{2}(\beta(t))\right)+1 \\
0=-y_{1}(t)-0.2 \sin ^{2}\left(x_{1}(t)\right)+0.3 \sin ^{2}\left(x_{1}(t)\right) \\
-0.2 \sin ^{2}\left(y_{1}(t)\right)+0.3 \sin ^{2}\left(y_{2}(t)\right)+1 \\
\frac{\mathrm{d} x_{2}}{\mathrm{~d} t}=-x_{2}(t)+0.005 \sin ^{2}\left(x_{1}(\beta(t))\right)+0.005 \sin ^{2}\left(x_{2}(\beta(t))\right) \\
+0.005 \sin ^{2}\left(y_{1}(\beta(t))\right)+0.005 \sin ^{2}\left(y_{2}(\beta(t))\right)+1 \\
0=-y_{2}(t)+0.2 \sin ^{2}\left(x_{1}(t)\right)+0.3 \sin ^{2}\left(x_{2}(t)\right) \\
+0.2 \sin ^{2}\left(y_{1}(t)\right)+0.3 \sin ^{2}\left(y_{2}(t)\right)+1
\end{array}\right.
$$

where $\left\{\alpha_{k}\right\}=\{\kappa k / 4\},\left\{\eta_{k}\right\}=\{\kappa k+1 / 8\}, k \in N$ and $\kappa \in N$. When $t \in\left[\alpha_{k}, \alpha_{k+1}\right)$, fix argument function $\beta(t)=\eta_{k}$.

Consider an undisturbed system,

$$
\left\{\begin{array}{l}
\frac{\mathrm{d} x_{1}}{\mathrm{~d} t}=-x_{1}(t)-0.005 \sin ^{2}\left(x_{1}(t)\right)+0.005 \sin ^{2}\left(x_{2}(t)\right) \\
-0.005 \sin ^{2}\left(y_{1}(t)\right)+0.005 \sin ^{2}\left(y_{2}(t)\right)+1 \\
0=-y_{1}(t)-0.2 \sin ^{2}\left(x_{1}(t)\right)+0.3 \sin ^{2}\left(x_{2}(t)\right) \\
-0.2 \sin ^{2}\left(y_{1}(t)\right)+0.3 \sin ^{2}\left(y_{2}(t)\right)+1 \\
\frac{\mathrm{d} x_{2}}{\mathrm{~d} t}=-x_{2}(t)+0.005 \sin ^{2}\left(x_{1}(t)\right)+0.005 \sin ^{2}\left(x_{2}(t)\right) \\
+0.005 \sin ^{2}\left(y_{1}(t)\right)+0.005 \sin ^{2}\left(y_{2}(t)\right)+1 \\
0=-y_{2}(t)+0.2 \sin ^{2}\left(x_{1}(t)\right)+0.3 \sin ^{2}\left(x_{2}(t)\right) \\
+0.2 \sin ^{2}\left(y_{1}(t)\right)+0.3 \sin ^{2}\left(y_{2}(t)\right)+1
\end{array}\right.
$$

Based on a lot of the available conclusions, when $l=1.2, \varsigma=0.9$, system (38) is globally exponentially stable. Fix that $\eta>1=\ln (1.2) / 0.9=0.2026$, and here $L_{i}^{1}=L_{i}^{2}=L_{i}^{3}=L_{i}^{4}=1$. Then,

$$
\begin{aligned}
& m_{1}=\max _{1 \leq i \leq n}\left\{c_{i}+\rho L_{i}^{2} \sum_{j=1}^{n}\left|b_{j i}\right|\right\}=1.01 \\
& m_{2}=\max _{1 \leq i \leq n}\left\{L_{i}^{2} \sum_{j=1}^{n}\left|a_{j i}\right|\right\}=1.01
\end{aligned}
$$

Substituting the above data into (30) and (31) yields

$$
\breve{x}[0.01+1.01(1+0.01 \breve{x}) \exp 0.01 \breve{x}]=1,
$$

and

$$
\begin{aligned}
& 1.2 \exp \{-0.9(1-\hat{x})\}+0.01(1+[1-0.01 \hat{x}-1.01 \hat{x} \\
& \left.(1+0.01 \hat{x}) \exp \{1.01 \hat{x}\}]^{-1}\right) \frac{1.2}{0.9} \\
& \quad \times \exp \left\{2\left(1.01+2 \times 0.01+[1-0.01 \hat{x}-1.01 \hat{x}(1+0.01 \hat{x}) \exp \{1.01 \hat{x}\}]^{-1} 0.01\right)\right\}=1 .
\end{aligned}
$$




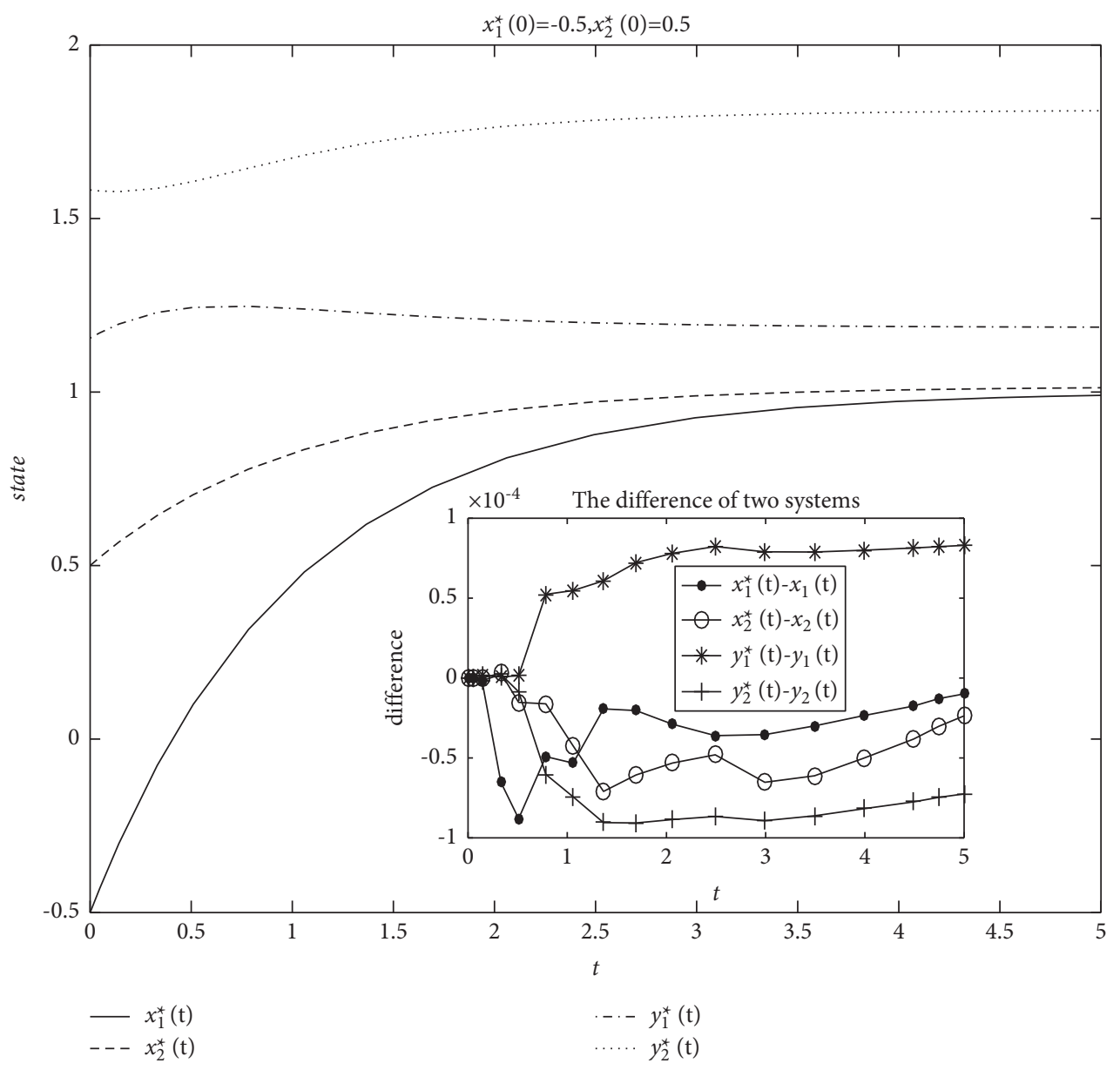

Figure 1: States $\left(x_{1}(t), y_{1}(t)\right),\left(x_{2}(t), y_{2}(t)\right)$ of models (57) and (58) with consistent initial values $x_{1}=-0.5, x_{2}=0.5$ of differential variables.

By solving the above transcendental equation, we can get $\mathscr{I}^{1}=0.5561$ and $\mathscr{I}^{2}=0.6778$. According to Theorem 1 , when $\alpha<\min \left(\eta / 2, \mathscr{I}^{1}, \mathscr{J}^{2}\right)$, that is, $\alpha<0.5$, then system (4) is globally exponentially stable.

Example 2. Consider a one-dimensional DAS,

$$
\left\{\begin{array}{l}
\frac{\mathrm{d} u(t)}{\mathrm{d} t}=-1.1 u(t)+0.05 \tanh (u(t))+0.05 \tanh (v(t)) \\
+1 \\
0=-v(t)+0.4 \tanh (u(t))+0.6 \tanh (v(t)) \\
+1 .
\end{array}\right.
$$

According to the current conclusions, when $l=1.2, \varsigma=1$, system (62) is globally exponentially stable.
The deviation variable and stochastic disturbance are applied to system (62) to obtain the following system:

$$
\left\{\begin{array}{l}
\mathrm{d} x(t)=[-1.1 x(t)+0.001 \tanh (x(\beta(t))) \\
+0.099 \tanh (y(t))+1] \mathrm{d} t+\sigma x(t) \mathrm{d} W(t), \\
0=-y(t)+0.4 \tanh (x(t))+0.6 \tanh (y(t))+1,
\end{array}\right.
$$

where $\beta(t)=\eta_{k}, o=0.3>\ln (l) / \varsigma=\ln (1.2) / 1=0.1823, t \epsilon$ $\left[\alpha_{k}, \alpha_{k+1}\right), k \in N, t \in R^{+},{ }_{t} W(t)$ compliance normal distribution as $W(t) \sim N(0, \stackrel{t}{\sigma})$.

$$
\begin{aligned}
& m_{1}=\max _{1 \leq i \leq n}\left(c_{i}+\rho L_{i}^{2} \sum_{j=1}^{2}\left|b_{j i}\right|\right)=1.001, \\
& m_{2}=\max _{1 \leq i \leq n}\left(L_{i}^{1} \sum_{j=1}^{2}\left|a_{j i}\right|\right)=0.001 .
\end{aligned}
$$

Substituting the above data into (27), the following equation can be obtained: 


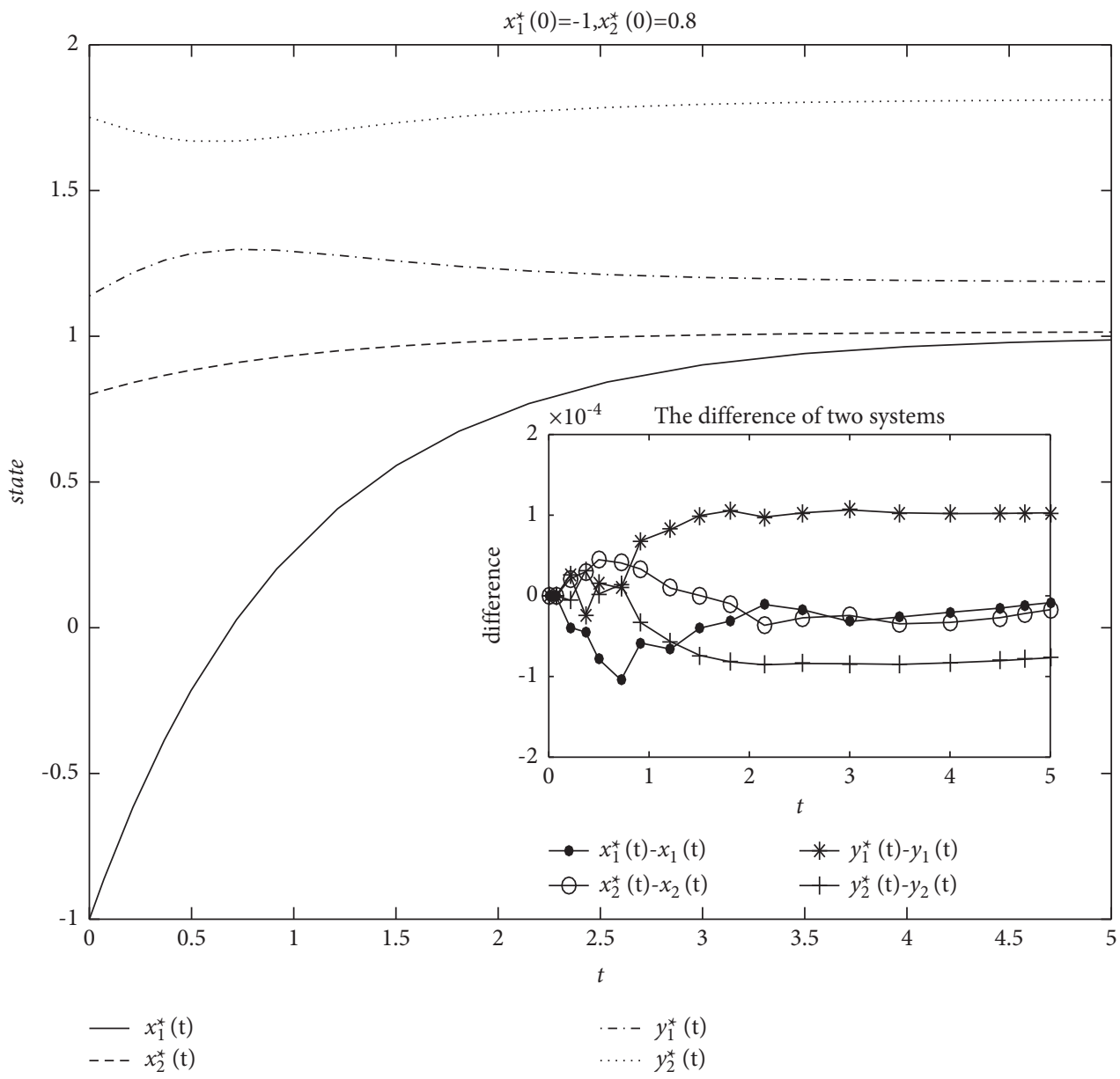

Figure 2: States $\left(x_{1}(t), y_{1}(t)\right),\left(x_{2}(t), y_{2}(t)\right)$ of models (57) and (58) with consistent initial values $x_{1}=0, x_{2}=0.8$ of differential variables.

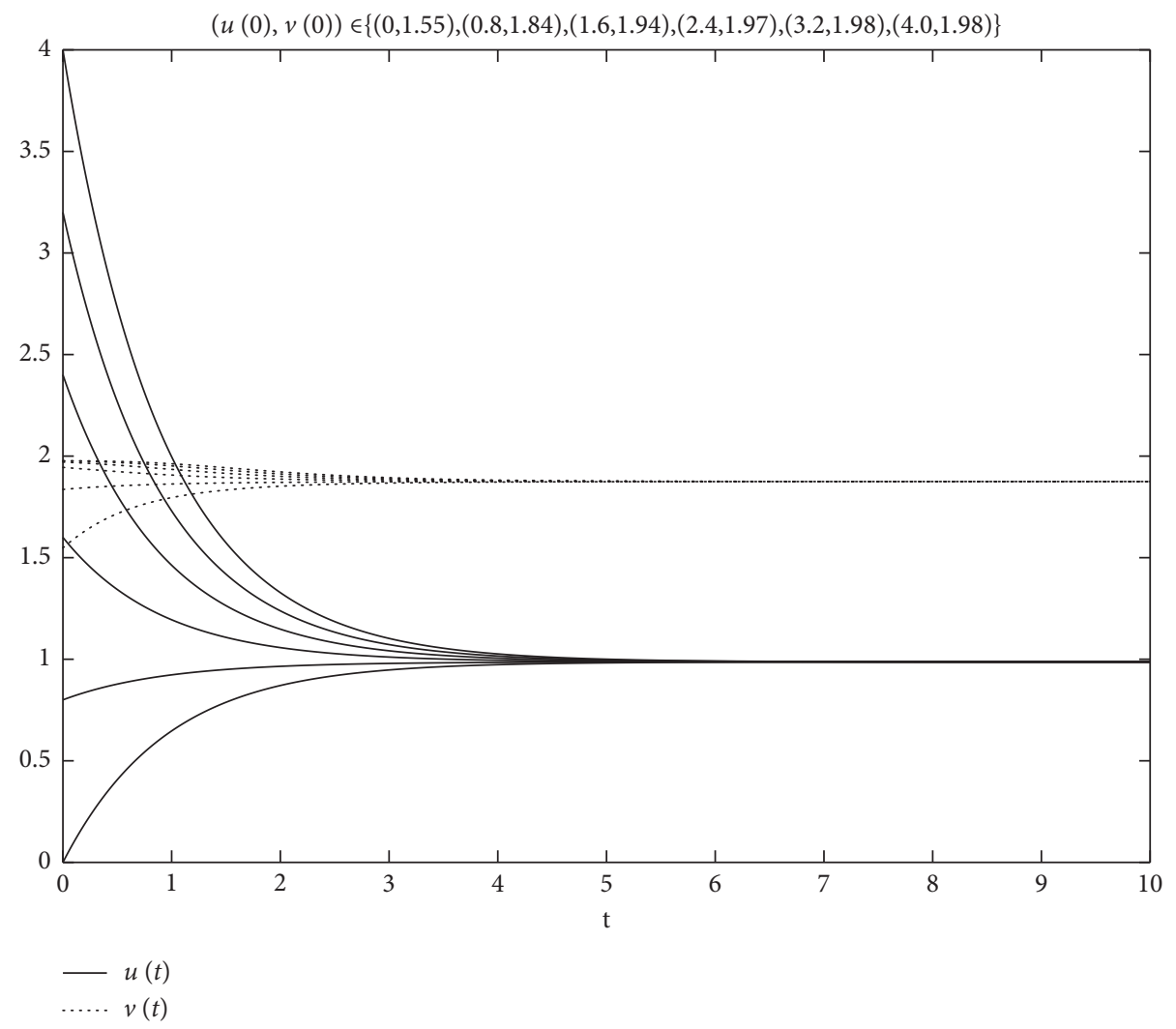

Figure 3: States $u(t)$ and $v(t)$ of model (36) with six groups of constant initial values. 

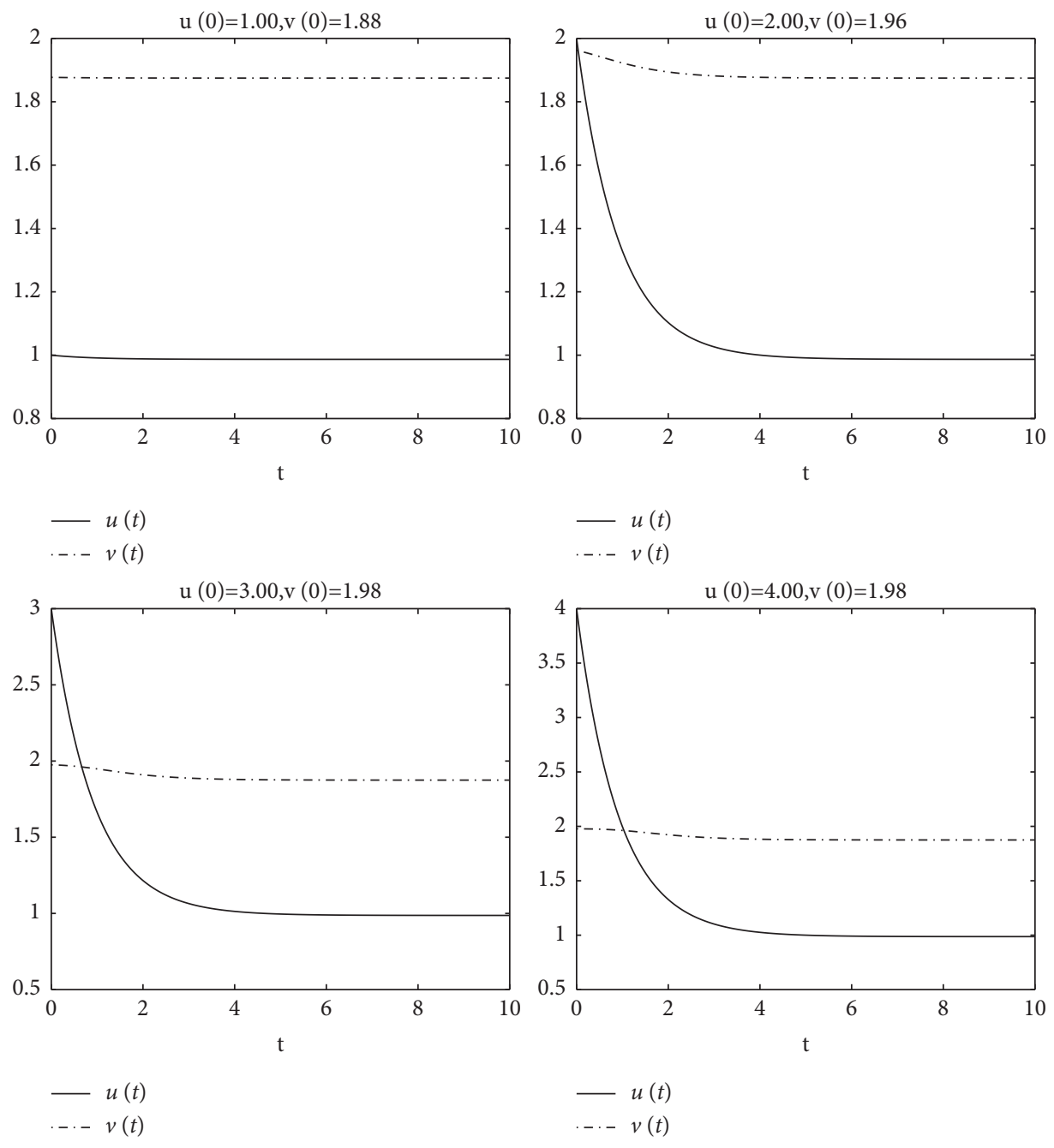

Figure 4: States $u(t)$ and $v(t)$ of model (62) with different consistent initial values alone.

$$
\begin{aligned}
& 2 \times 1.2 \exp \{-3 \times 0.3\}+2\left[288 \times 0.001^{2} \times 0.3+6 \hat{y}^{2}\right] \\
& \frac{2 \times 0.3 \times 1.2}{1} \times \exp \left\{\left[12 \times 0.3 \times\left(1.001^{2}\right.\right.\right. \\
& \left.\left.\left.+2 \times 0.001^{2}\right)+288 \times 0.001^{2} \times 0.3+6 \hat{y}^{2}\right]\right\}=1 .
\end{aligned}
$$

Therefore, $\bar{\sigma}=0.0152$, since $|\sigma|<\bar{\sigma} / \sqrt{6}$, then $|\sigma|<0.0062$. Subjecting $\sigma$ to (30), we can obtain that

$$
\begin{aligned}
& 2 \exp -3(0.3-\breve{y}) \\
& \quad+2\left[96 \times 0.001^{2} \times 0.3+0.0062^{2}\right] \frac{0.3 \times 1.2}{1} \\
& \exp \left\{12 \times 0.3 \times\left(1.1^{2}+2 \times 0.001^{2}\right)\right. \\
& \left.\quad+96 \times 0.001^{2} \times 0.3+0.0062^{2}\right\}=1 .
\end{aligned}
$$

Then, it is easy to get $\mathscr{I}^{1}=0.0677, \alpha<\min \left(o / 2, \mathscr{I}^{1}\right)$, so $\alpha<0.0677$.
4.2. Simulation Results. Figures 1 and 2 show the stability characteristics of models (57) and (58) with different initial values. A contrast diagram of the convergence difference between the undisturbed and disturbed systems is given.

Figures 3 and 4 show the stability characteristics of model (62) with different initial values. Figure 3 shows that differential variables and algebraic variables converge to a point starting from different initial values, and the initial values of the algebraic variables are dependent on the differential variables, which demonstrates the integrity of the system. Figure 4 shows the stability of the system with different initial values.

Figure 5 shows the stability of system (63) in the case of deviations and SDs. A comparison of 9 groups of solutions is 


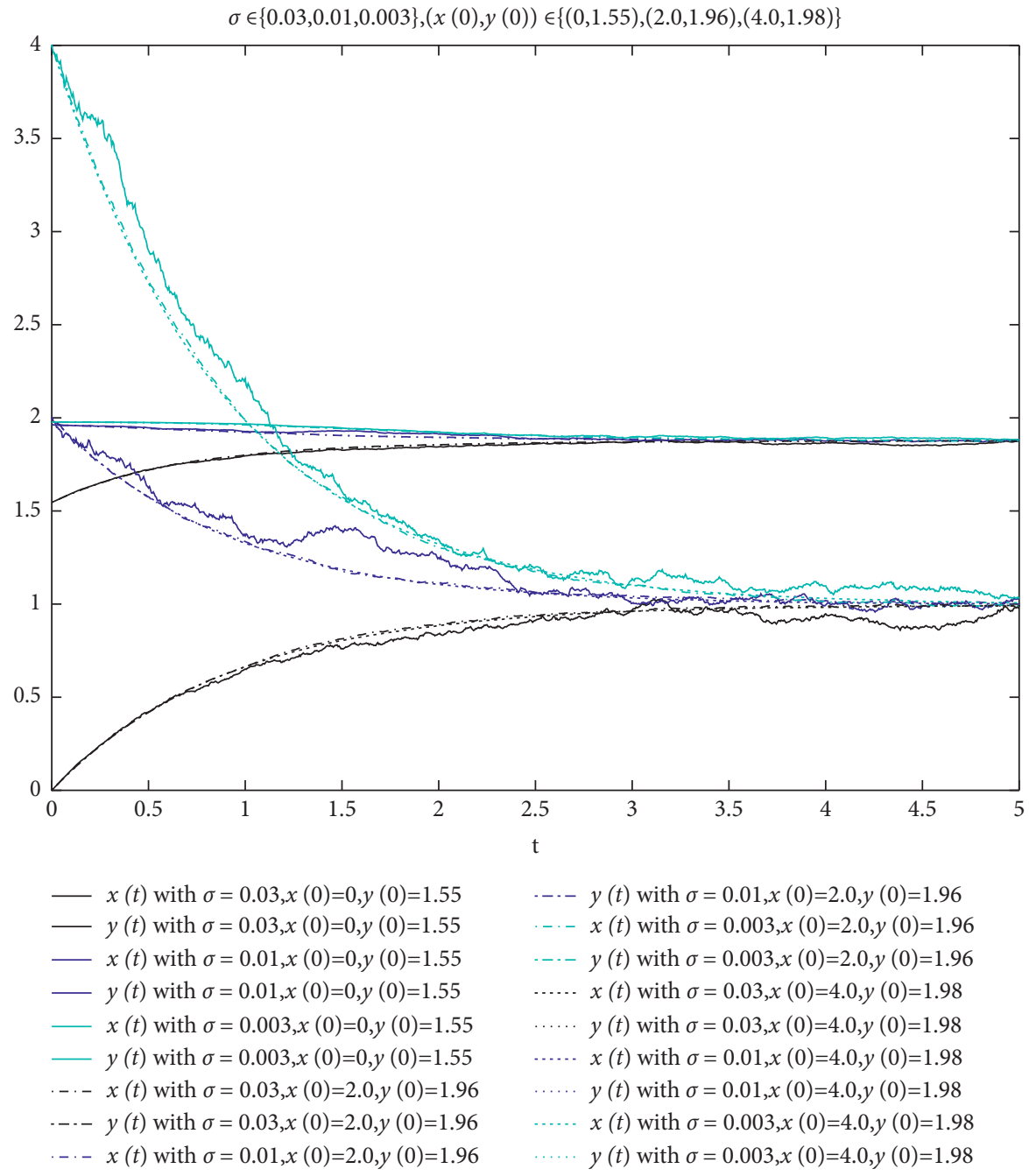

Figure 5: States $x(t)$ and $y(t)$ of model (38) with nine groups of consistent initial values.

given in the figure. In this paper, it is assumed that $\sigma$ obeys a normal distribution. This figure illustrates a simulation based on the Euler method, and the step size is 0.005 .

\section{Conclusion}

This article is dedicated to the study of the robustness of the global exponential stability and mean-square exponential stability of nonlinear DASs with DAs and SDs. This paper describes DASs with DAs and SDs for the first time, and the results provide the minimum upper bounds of the deviation interval and noise intensity, which make the system stable. This paper presents traditional Hopfield neural networks as DASs, which have a certain practical significance for enriching the theory of neural networks. Furthermore, this article derives that the interfered system is still stable under a certain degree of interference on the premise that the undisturbed system is stable. The analysis method is very novel, and the system model considers a more general situation. The conditions that ensure the DASs are index-1 are strong.
Whether this approach can be extended to systems with high indices is a topic for future work. The synchronization and multistability of such models will be explored.

\section{Data Availability}

The data used in the model simulation are generated by computer according to the mathematical description in the model based on the Euler method, with the step size of 0.005 .

\section{Conflicts of Interest}

The authors declare that they have no conflicts of interest.

\section{Acknowledgments}

This work was supported by the National Natural Science Foundation of China (no. 72071092) and open fund of Hubei Key Laboratory of Metallurgical Industry Process System Science (nos. Y201720 and Y202005). 


\section{References}

[1] L. Hu and X. Li, "Adaptive neural finite-time stabilisation for a class of p-normal form nonlinear systems with unknown virtual control coefficients," International Journal of Control, vol. 94, no. 5, pp. 1386-1401, 2019.

[2] Y.-Q. Han, S.-L. Zhu, D.-Y. Duan, L. Chu, P.-C. Xiong, and S.-G. Yang, "Observer-based adaptive neural tracking control for a class of stochastic nonlinear systems," International Journal of Control, vol. 94, no. 5, pp. 1344-1354, 2019.

[3] C. Wang, Y. Wu, F. wang, and Y. Zhao, "TABLF-based adaptive control for uncertain nonlinear systems with timevarying asymmetric full-state constraints," International Journal of Control, vol. 94, no. 5, pp. 1238-1246, 2019.

[4] Q. Quan and K.-Y. Cai, "Repetitive control for nonlinear systems: an actuator-focussed design method," International Journal of Control, vol. 94, no. 5, pp. 1225-1237, 2021.

[5] X. Li, X. Lin, and Y. Zou, "Output feedback stabilization for planar switched nonlinear systems with asymmetric output constraints," Nonlinear Analysis: Hybrid Systems, vol. 40, p. 101005, 2021.

[6] S. Yan, W. Sun, and F. He, "Adaptive output feedback tracking control for a class of nonlinearly parameterised uncertain systems," International Journal of Control, vol. 94, no. 5, pp. 1174-1187, 2021.

[7] M. E. Koksal, "Commutativity of systems with their feedback conjugates," Transactions of the Institute of Measurement and Control, vol. 41, no. 3, pp. 696-700, 2019.

[8] S. Dong, G. Chen, M. Liu, and Z.-G. Wu, "Robust adaptive Ho control for networked uncertain semi-Markov jump nonlinear systems with input quantization," Science China Information Sciences, vol. 65, no. 8, pp. 1174-1187, 2022.

[9] H. Wu, S. Liu, C. Cheng, and C. Du, "Observer based direct adaptive fuzzy second-order-like sliding mode control for unknown nonlinear systems," Proceedings of the Institution of Mechanical Engineers-Part E: Journal of Process Mechanical Engineering, vol. 235, no. 2, pp. 197-207, 2021.

[10] L. Nisse and A. Bouaziz, "Existence and stability of the solutions for systems of nonlinear fractional differential equations with deviating arguments," Advances in Difference Equations, vol. 2014, no. 1, pp. 1-10, 2014.

[11] Q. Xi and X. Liu, "Finite-time stability and controller design for a class of hybrid dynamical systems with deviating argument," Nonlinear Analysis: Hybrid Systems, vol. 39, p. 100952, 2021.

[12] N. Durga and P. Muthukumar, "Optimal control of fractional neutral stochastic differential equations with deviated argument governed by Poisson jumps and infinite delay," Optimal Control Applications and Methods, vol. 40, no. 5, pp. 880-899, 2019.

[13] W. Cheng, A. Wu, J.-E. Zhang, and B. Li, "Outer-synchronization of fractional-order neural networks with deviating argument via centralized and decentralized data-sampling approaches," Advances in Difference Equations, vol. 2019, no. 1, 2019.

[14] J. Chen and X. Qin, "Monotone iterative method for two types of integral boundary value problems of a nonlinear fractional differential system with deviating arguments," Journal of Mathematics, vol. 2021, Article ID 6650811, 8 pages, 2021.

[15] Z. Y. Zhang, R. H. Feng, I. Jadlovska, and Q. M. Liu, "Oscillation criteria for third-Order nonlinear neutral dynamic equations with mixed deviating arguments on time scales," Mathematics, vol. 94, no. 5, pp. 1238-1246, 2021.
[16] Z. Dai, L. Xu, and S. S. Ge, "Attracting sets of discrete-time Markovian jump delay systems with stochastic disturbances via impulsive control," Journal of the Franklin Institute, vol. 357, no. 14, pp. 9781-9810, 2020.

[17] X. M. Zhang and H. N. Wu, " $H_{\infty}$ boundary control for a class of nonlinear stochastic parabolic distributed parameter systems," International Journal of Robust and Nonlinear Control, vol. 29, no. 14, pp. 4665-4680, 2019.

[18] C.-Y. Chen, W.-H. Gui, Z.-H. Guan, R.-L. Wang, and S.-W. Zhou, "Adaptive neural control for a class of stochastic nonlinear systems with unknown parameters, unknown nonlinear functions and stochastic disturbances," Neurocomputing, vol. 226, no. 22, pp. 101-108, 2017.

[19] Y. Ren, W. Wang, W. Zhou, and M. Shen, "Stochastic incremental $H_{\infty}$ control for discrete-time switched systems with disturbance dependent noise," Information Sciences, vol. 513, pp. 519-535, 2020.

[20] J.-E. Zhang, "Robustness analysis of global exponential stability of nonlinear systems with deviating argument and stochastic disturbance," IEEE Access, vol. 5, pp. 13446-13454, 2017.

[21] K. N. Akpinar, S. Genc, and S. Karagol, "Chest X-ray abnormality detection based on SqueezeNet," in Proceedings of the 2020 International Conference on Electrical, Communication, and Computer Engineering (ICECCE), Istanbul, Turkey, June 2020.

[22] S. Genc, K. N. Akpinar, and S. Karagol, "Automated abnormality classification of chest radiographs using MobileNetV2," in Proceedings of the 2020 International Congress on Human-Computer Interaction, Optimization and Robotic Applications (HORA), Ankara, Turkey, June 2020.

[23] G. Koppe, H. Toutounji, P. Kirsch, S. Lis, and D. Durstewitz, "Identifying nonlinear dynamical systems via generative recurrent neural networks with applications to fMRI," PLoS Computational Biology, vol. 15, no. 8, pp. e1007263-35, 2019.

[24] E. Yuksel, D. Soydaner, and H. Bahtiyar, "Nuclear binding energy predictions using neural networks: application of the multilayer perceptron," International Journal of Modern Physics E, vol. 30, no. 03, p. 2150017, 2021.

[25] S. Genc and S. Karagol, "Fuzzy logic application in DGA methods to classify fault type in power transformer," in Proceedings of the 2020 International Congress on HumanComputer Interaction, Optimization and Robotic Applications (HORA), pp. 1-4, Ankara, Turkey, June 2020.

[26] E. Guney, S. Karag, and M. Demir, "A comparative real-time speed control of PMSM with fuzzy logic and ANN based vector controller," Sirnak Universitesi Fen Bilimleri Dergisi, vol. 1, no. 1, pp. 123-143, 2019.

[27] A. Kumar, Control of Nonlinear Differential Algebraic Equation Systems with Applications to Chemical Processes, CRC Press, Boca Raton, FL, USA, 1999.

[28] A. González-Zumba, P. Fernández-de-Córdoba, J.-C. Cortés, and V. Mehrmann, "Stability assessment of stochastic differential-algebraic systems via Lyapunov exponents with an application to power systems," Mathematics, vol. 8, no. 9, p. 1393, 2020.

[29] A. K. W. Navarro and V. S. Vassiliadis, "Computer algebra systems coming of age: dynamic simulation and optimization of DAE systems in Mathematica," Computers \& Chemical Engineering, vol. 62, no. 5, pp. 125-138, 2014.

[30] J. Åslund and E. Frisk, "An observer for non-linear differential-algebraic systems," Automatica, vol. 42, no. 6, pp. 959-965, 2006. 
[31] Q. Lan, L. Sun, J. Liang et al., "Sampled-data stabilization of a class of nonlinear differential algebraic systems via partialstate and output feedback," Journal of the Franklin Institute, vol. 357, no. 8, pp. 4742-4758, 2020.

[32] P. Di Franco, G. Scarciotti, and A. Astolfi, "Stability of nonlinear differential-algebraic systems via additive identity," IEEE/CAA Journal of Automatica Sinica, vol. 7, no. 4, pp. 929-941, 2020.

[33] B. Shi, H. Peng, X. Wang, W. Zhong, L. Gao, and J. Fottner, "A symplectic indirect approach for a class of nonlinear optimal control problems of differential-algebraic systems," International Journal of Robust and Nonlinear Control, vol. 31, no. 7, pp. 2712-2736, 2021. 